\title{
Síndrome coronario asociado al consumo de cocaína: serie de casos
}

\section{Acute coronary syndrome associated with the consumption of cocaine: case series}

\author{
Nicolás Wilches-Rivas ${ }^{1}$, Marie Claire Berrouet-Mejía ${ }^{2}$
}

\section{RESUMEN:}

El consumo de cocaína continúa siendo un problema social importante con un impacto significativo en la salud de la población expuesta, en un alto porcentaje integrada por adolescentes y adultos jóvenes. Entre las complicaciones cardiovasculares asociadas al consumo de cocaína se

\section{ABSTRACT:}

Cocaine continues to be a major social problem with a significantimpact on the health of the exposedpopulation, a high proportion composed of adolescents and young adults. Among the cardiovascular complications associated with cocaine use are the acute coronarysyndrome, sudden death,

\section{Historial del artículo:}

Fecha de recepción: 30/12/2019

Fecha de aceptación: 13/05/2020

1 Hospital San Vicente Fundación, Urgentólogo. Medico Universidad de Antioquia. Especialista en Medicina de Urgencias Universidad CES.

2 Universidad CES. Toxicóloga clínica Universidad de Antioquia. Toxioóloga Hospital General de Medellín y Clínica SOMA. Investigadora grupo CES-HGM.

Correspondencia: Marie Claire Berrouet Mejía, Toxicóloga Clínica. Dirección: Cl. 10a \#22 - 04, Universidad CES. Medellín, Antioquia. Teléfono: 3847300 ext. 1847. Correo Electrónico: mcberrouet@hotmail.com

Como citar este artículo: Wilches-Rivas N, Berrouet-Mejía M. Síndrome coronario asociado al consumo de cocaína: serie de casos. Revista de la Facultad de Ciencias de la Salud de la Universidad del Cauca. 2020; 22(2):43-49. https://doi.org/10.47373/rfcs.2020.v22.1311 
encuentran el síndrome coronario agudo, la muerte súbita, las arritmias, el edema pulmonar, la miocarditis, la endocarditis, la disección aórtica, las crisis hipertensivas y el accidente cerebrovascular. El objetivo de esta serie de casos es presentar tres pacientes menores de 40 ańos con antecedentes como consumo crónico de cocaína, tabaco y alcohol, quienes ingresan a un servicio de urgencias sin toxidrome adrenérgico pero con manifestaciones clínicas, biomarcadores y electrocardiograma sugestivos de infarto agudo de miocardio tipo 1 asociado al consumo de cocaína.

Palabras Claves: Sindrome Coronario Agudo, Cocaína, Conducta del Adolescente, AdultoJoven, Trombosis. (DeCS)

\section{INTRODUCCIÓN}

La Organización de las Naciones Unidas muestra los cambios en el consumo de sustancias psicoactivas durante el tiempo; desde el ańo 2013 empezó el reporte de cambios en los patrones de consumo a nivel mundial, con un aumento en el abuso de medicamentos recetados y de nuevas sustancias psicoactivas $(1,2)$.

Con respecto a las sustancias consumidas por colombianos, el último informe del Observatorio de Drogas reporta con mayor uso a la marihuana, la cocaína, el basuco y el éxtasis. Este informe también resalta la aparición de 28 nuevas sustancias psicoactivas, en contraste con 739 reportadas en el informe mundial de las Naciones Unidas para Colombia; adicionalmente, se indica que de cada cinco consumidores uno es mujer y el grupo etario de mayor consumo está entre los 18 y 24 ańos, con mayor riesgo de abuso y dependencia en los estratos socioeconómicos bajos (2).

La cocaína es un alcaloide deletéreo para todos los grupos de edad y existen ciertos factores de riesgo que aumentan la morbimortalidad asociada como el tabaquismo y el consumo de alcohol y otras sustancias de abuso, dentro de las que vale la pena resaltar la heroína (3-6).

El objetivo de esta serie de casos fue presentar la ocurrencia de complicaciones cardiovasculares -específicamente el síndrome coronario agudo (SCA)- en pacientes con consumo de cocaína, puesto que es una complicación que genera debate en su tratamiento agudo y crónico (6-8). arrhythmias, pulmonary edema, myocarditis, endocarditis, aortic dissection, hypertensive crisis and stroke. Our aim is topresent threepatients under 40 years of age with a history of chronic cocaine, tobacco and alcohol consumption who are admitted to the emergency room without adrenergic toxidrome, but with clinical findings with suspicion of type 1 acute myocardial infarction associated with cocaine use.

Key Words: Acute Coronary Syndrome, Cocaine, Adolescent Behavior, Young Adult, Thrombosis. (MeSH)

\section{DESCRIPCIÓN DEL CASO UNO}

Paciente de sexo masculino de 24 ańos, mestizo. Consultó al servicio de urgencias por presentar 2 horas de evolución con dolor precordial intenso que se manifestó a los 10 minutos del inicio de actividad física durante un partido de futbol. Las características del dolor fueron de inicio súbito, opresivo, irradiado a espalda, con una duración de 20 minutos y autolimitación con el reposo. El paciente relató tres episodios similares en el mes anterior que se desencadenaban con el ejercicio. Como antecedentes patológicos de importancia refirió que hace varios meses le diagnosticaron hipertensión arterial; sin embargo, no estaba medicado y era fumador de cigarrillos (10 paquetes/ ańo desde los 15 ańos).

Ingresó al servicio por sus propios medios, sin dolor precordial, con signos vitales presión arterial de 131/80 $\mathrm{mmHg}$, presión arterial media $97 \mathrm{mmHg}$, frecuencia cardiaca de 98 latidos por minuto, frecuencia respiratoria 28 por minuto, $\mathrm{SaO} 295 \%$. Como hallazgos al examen físico presentaba taquicardia con extrasístoles frecuentes, sin soplos, sin frote pericárdico; el resto del examen físico sin alteraciones. Se tomó electrocardiograma de 12 derivaciones con una velocidad de $25 \mathrm{~mm} / \mathrm{s}$ y amplitud de $1 \mathrm{mV}$ por $10 \mathrm{~mm}$ (Figura 1) en el cual se encontró taquicardia sinusal, elevación del segmento ST en las derivaciones V2-V3-V4-V5 y extrasístoles ventriculares en V1-V2-V3, sin otros hallazgos de relevancia. Se inició enfoque para SCA vs pericarditis aguda y se solicitaron paraclínicos que reportaron hemoglobina $15.3 \mathrm{mg} / \mathrm{dl}$, recuento de plaquetas 381.000 , leucocitos 19.000, neutrófilos 47\%, PCR $0.08 \mathrm{mg}$ / $\mathrm{dl}$, creatinina $0.7 \mathrm{mg} / \mathrm{dl}$ y troponina I $22.4 \mathrm{pg} / \mathrm{ml}$. 
Figura 1. Electrocardiograma de 12 derivaciones. Caso uno.

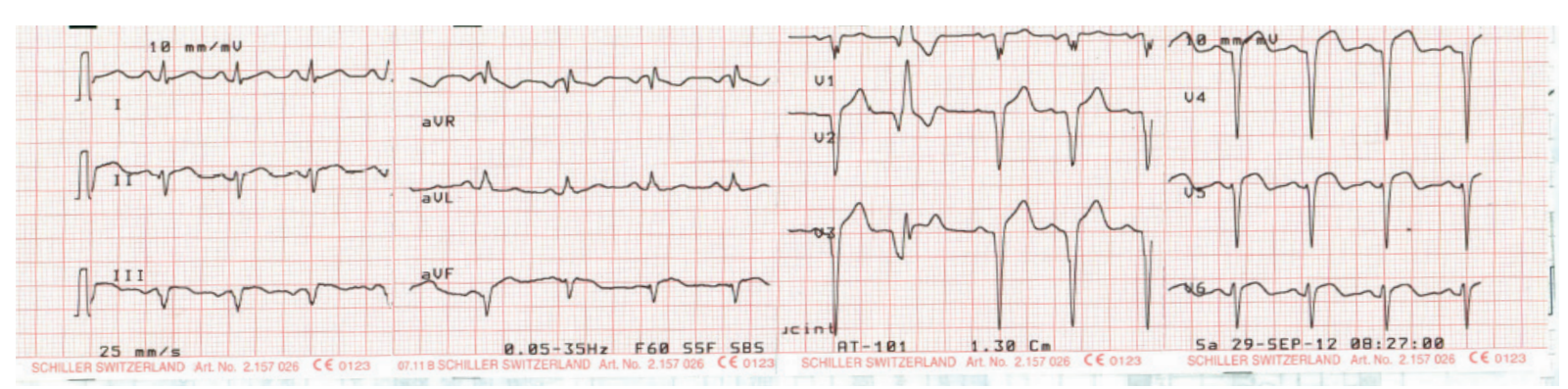

El paciente fue valorado por cardiología y posteriormente trasladado a sala de hemodinamia, previo manejo antiisquémico, donde encontraron ventriculografía izquierda con volúmenes moderadamente aumentados, gran área discinética anterior y balonamiento apical. Fracción de eyección del $35 \%$, coronaria izquierda con trombo en la descendente anterior. Se diagnosticó trombosis proximal de la descendente anterior con infarto agudo de miocardio (IAM) anterior extenso.

Posteriormente se interrogó al paciente quien comentó que el día anterior al inicio del cuadro clínico estuvo consumiendo cocaína. Cinco días después fue dado de alta con tratamiento farmacológico con ácido acetilsalicílico $100 \mathrm{mg} /$ día, clopidogrel $75 \mathrm{mg} /$ día, enalapril $20 \mathrm{mg} /$ día, atorvastatina $40 \mathrm{mg}$ / día y carvedilol 6,25mg/día.

\section{DESCRIPCIÓN DEL CASO DOS}

Paciente de sexo masculino de 35 ańos de edad quien como único antecedente manifestaba consumo de cocaína

Figura 2. Electrocardiograma de 12 derivaciones. Caso dos.

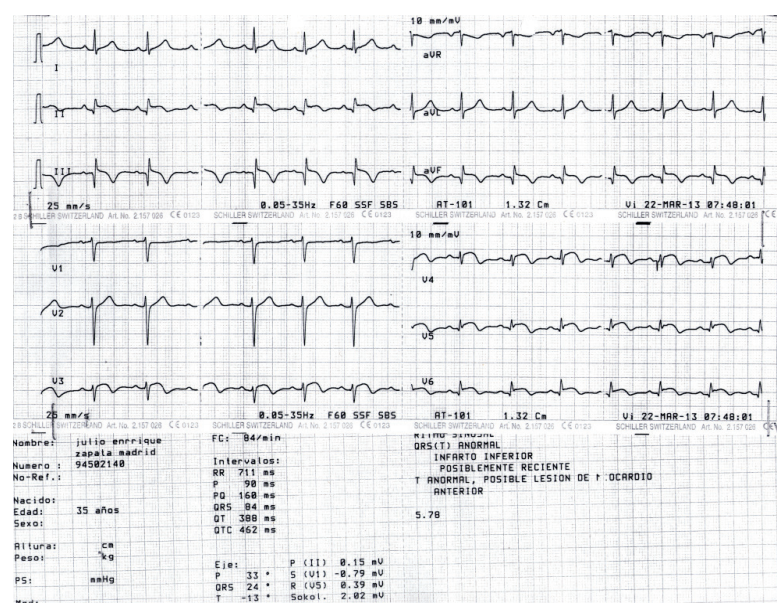

(aproximadamente 1 gramo al día por 10 ańos) y alcohol los fines de semana hasta la embriaguez. Consultó por dolor precordial opresivo de una hora de evolución asociado a disnea, diaforesis, náuseas y vómito. Se realizó electrocardiograma donde se evidenció elevación del segmento ST en pared anterior y las troponinas de $8 \mathrm{pg} /$ $\mathrm{ml}$ (rango normal de 0.020 y $0.029 \mathrm{pg} / \mathrm{ml}$ ). Ingresó en condiciones hemodinámicas estables y al examen físico con signos vitales presión arterial de 130/82 mmHg, presión arterial media $98 \mathrm{mmHg}$, frecuencia cardiaca 88 latidos por minuto, sin evidencia de alteraciones neurológicas. Se solicitó un nuevo electrocardiograma en donde encontraron elevación del segmento ST en pared ínfero-lateral DIIDIII-AVF-V5-V6 (Figura 2). Se inició tratamiento con cargas de ácido acetil salicílico, clopidogrel, enoxaparina y nitroglicerina.

Se comentó con cardiología intervencionista y se trasladó a coronariografía urgente que reportó hipocinesia posteroinferior, fracción de eyección de $50 \%$ en coronaria derecha de distribución dominante. Se evidenció gran trombo en tercio proximal de la misma arteria con flujo TIMI 3 (normal). Se confirmó enfermedad coronaria tipo IAM inferior. El paciente fue dado de alta 24 horas después del procedimiento con indicaciones de ácido acetilsalicílico $100 \mathrm{mg} /$ día, clopidogrel 75mg/día, enalapril $20 \mathrm{mg} /$ día, atorvastatina $40 \mathrm{mg} /$ día, metoprolol 100mg/día.

\section{DESCRIPCIÓN DEL CASO TRES}

Paciente de sexo femenino de 39 ańos, habitante de calle con antecedente de consumo de cigarrillo, alcohol y clorhidrato de cocaína diario. Dieciocho horas posteriores a la ingesta de 10 gramos de cocaína consultó por dolor opresivo, retroesternal que se irradió a región precordial anterior, de inicio súbito en reposo y con duración mayor a 20 minutos. Este dolor se repitió varias veces en el día mientras continuó el consumo de clorhidrato de cocaína y 
pasta básica de coca en menor cantidad. En la anamnesis refirió que el consumo de cocaína siempre le desencadenaba malestar, pero el dolor que la llevó a consultar fue el peor de toda su vida y se acompańó con sensación de muerte, debilidad, diaforesis y náuseas.

Al examen físico se encontraba en malas condiciones generales: diaforética, pálida, ansiosa, pero sin disnea. Frecuencia cardiaca de 72 latidos por minuto, presión arterial de 130/90 $\mathrm{mmHg}$, frecuencia respiratoria de 14 respiraciones por minuto, saturación de oxígeno $87 \%$ con Fi02 $21 \%$, temperatura $35.5^{\circ} \mathrm{C}$. Dolor precordial que califió como 10/10 en escala numérica del dolor sin evidencia de alteraciones neurológicas y sin otros hallazgos patológicos en el sistema cardiovascular.

Se le realizó electrocardiograma donde se encontraron hallazgos sugestivos de IAM inferior, dado por elevación del segmento ST convexa en las derivaciones DII, DIII y aVF, además de presencia de ritmo de fibrilación auricular (Figura 3). No pudo ser remitida para intervención coronaria percutánea primaria y se decidió estrategia de tratamiento con terapia de reperfusión primaria con fibrinolítico (pensando en una intervención coronaria percutánea rutinaria temprana en caso de fibrinólisis exitosa), utilizando tenecteplase $40 \mathrm{mg}$ en bolo intravenoso.

Se utilizó como método de anticoagulación reversible heparina no fraccionada (dosis de 60 unidades por kilogramo intravenoso iniciales, seguido por infusión de $12 \mathrm{u} / \mathrm{kg} / \mathrm{h}$ ). Se indicó antiagregación dual con ácido acetilsalicílico y clopidogrel a dosis usuales. Se inició infusión de nitroglicerina iniciando a $0.25 \mathrm{mcg} / \mathrm{kg} / \mathrm{min}$. Durante la fase aguda no se iniciaron beta-bloqueadores; el procedimiento fue exitoso y se continuó con monitorización.

\section{DISCUSIÓN}

El clorhidrato de cocaína, extraído de la planta Erythroxylon coca, es una de las formas como se consume este alcaloide. Su absorción es rápida por ser una sustancia liposoluble y puede darse a través de varias vías de administración. Alcanza efectos rápidos (menos de un minuto) con una duración variable entre 30 y 120 minutos (6-9).

Los mecanismos de toxicidad del clorhidrato de cocaína se explican por la estimulación de los receptores adrenérgicos $\alpha$ y $\beta$ y la inhibición de la recaptación de catecolaminas. Con respecto a los metabolitos, es importante profundizar en el cocaetileno, producto de la coingesta de cocaína con alcohol pues este incrementa hasta 20 veces el riesgo de muerte súbita y vasoespamo (7-9).

Las complicaciones asociadas al consumo de cocaína se pueden dividir según sistemas en: neurológicas, cardiovasculares y respiratorias principalmente. Las dos primeras de gran importancia por la morbimortalidad asociada. Dentro de las cardiovasculares están el SCA, muerte súbita, arritmias, edema pulmonar, miocarditis, endocarditis, disección aórtica, hipertensión arterial y crisis hipertensivas (10-14).

Adicional a los mecanismos anteriormente expresados, es importante resaltar que la isquemia miocárdica está explicada por desbalance entre la entrega y demanda de oxígeno en el miocardio, un aumento en la producción de sustancias vasoconstrictoras como la endotelina y disminución en sustancias vasodilatadoras como el óxido nítrico (por inhibición del óxido nítrico sintasa) (10,13-15). También se ha reportado incremento del inhibidor del activador del plasminógeno y un aumento en los procesos de activación y agregación plaquetaria, lo cual explica los procesos de aterosclerosis acelerada (16).

Figura 3. Electrocardiograma de 12 derivaciones. Caso tres.

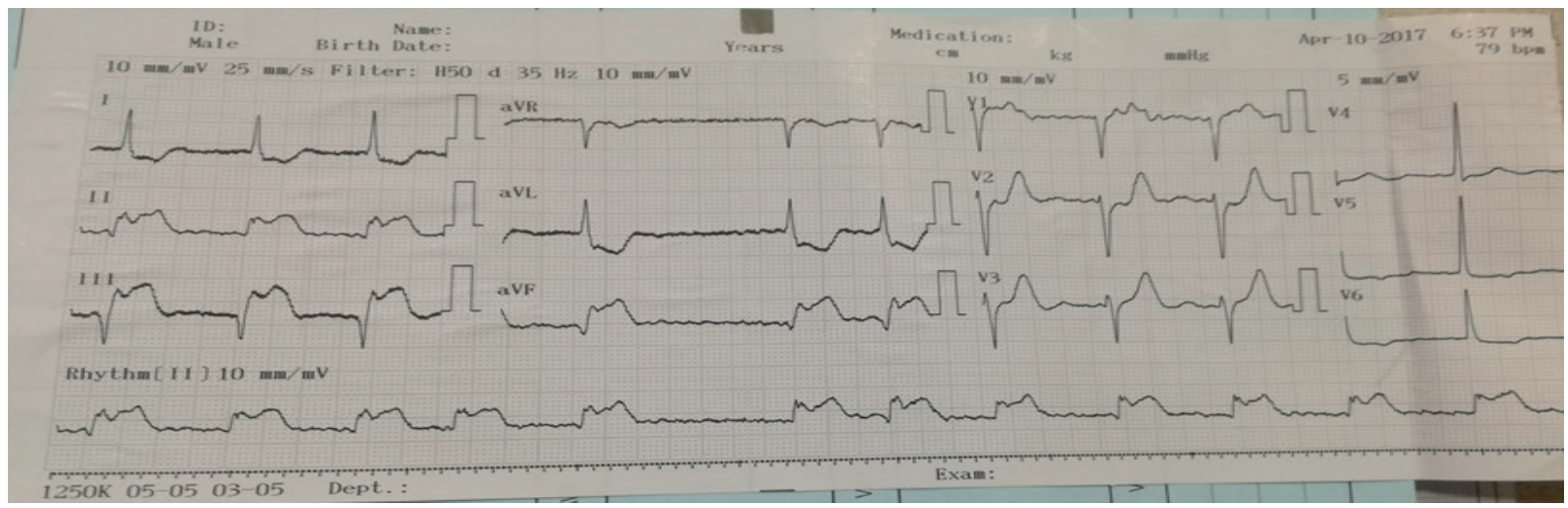


Las arritmias asociadas con la cocaína no están solo relacionadas a la hiperactividad adrenérgica, sino también al bloqueo de canales de sodio. Como consecuencia se puede observar desde taquicardia sinusal hasta arritmias ventriculares $(17,18)$.

Hasta un $15 \%$ de los pacientes que consultan por dolor torácico asociado al consumo de cocaína tienen un SCA. El consumo de este alcaloide aumenta el riesgo de sufrir un síndrome coronario agudo reportado en algunos estudios con un odds ratio [OR] 3.8 a $6.9(11,19)$. El riesgo de un IAM se incrementa hasta 24 veces en la primera hora después del consumo de cocaína; este riesgo es independiente de dosis, vía de utilización y frecuencia de consumo y el SCA se puede presentar en consumidores regulares o con el primer consumo (19-21).

En el abordaje del paciente con consumo agudo de cocaína y dolor torácico, en quien se sospecha un SCA, es importante establecer el tiempo entre el consumo y el evento el cual es generalmente corto, aunque existen reportes de hasta dos semanas luego de la última exposición. Entre las ayudas diagnósticas fundamentales están el electrocardiograma y la troponina I. El electrocardiograma puede ser difícil de interpretar por trastornos de repolarización temprana en pacientes jóvenes, en este escenario tiene una sensibilidad del $36 \%$ y especificidad del $90 \%$, con un valor predictivo positivo del 18\% y un valor predictivo negativo del 96\% (20-23).

La elevación de las enzimas creatinfosfokinasa y su fracción MB (CPK y CK MB) es común en pacientes consumidores y puede explicarse por otras causas por tanto se prefiere como herramienta diagnostica la troponina I que es más específica $(20,23-25)$.

Las benzodiacepinas, principalmente las de vida media larga como el diazepam se recomiendan de forma precoz por vía parenteral. El uso de las mismas se asocia a liberación de GMP cíclico y a una mejoría del dolor torácico, disminución de la frecuencia cardiaca y las cifras de presión arterial. Además de lo anterior, por su efecto gabaérgico, favorecen la sedación y tienen una recomendación IB $(14,25,26)$. El ácido acetilsalicílico está justificado por su efecto antiagregante plaquetario y tiene una recomendación IC (10).

Respecto a los vasodilatadores, en la literatura básicamente se mencionan dos: la nitroglicerina y el nitroprusiato de sodio, siendo más utilizada la primera. Este agente vasodilatador aumenta el flujo coronario por vasodilatación de los vasos epicárdicos y disminuye la compresión sistólica de los vasos coronarios. Su recomendación es IC (10). El uso de otros medicamentos como calcio antagonistas, específicamente verapamilo y fentolamina por vía parenteral, tiene una recomendación IIb/C (10).

Frente a la terapia de reperfusión, la estrategia recomendada es la angioplastia primaria. Esta estrategia ha probado ser superior a la trombólisis en pacientes con diagnóstico de IAM con elevación del ST; sin embargo, la terapia fibrinolítica debe considerarse en los pacientes a quienes no puede accederse de manera oportuna a un manejo intervencionista dentro de los tiempos establecidos (10).

Frente al uso de beta-bloqueadores en el SCA asociado a cocaína, en los últimos ańos se ha generado una gran controversia. El riesgo de esta familia de medicamentos en el SCA asociado al consumo de cocaína, es favorecer el estímulo de receptores alfa. Por otra parte, el uso de beta-bloqueadores puede reducir el riesgo de re-infarto y las arritmias ventriculares en contexto de IAM. Ya hay estudios que plantean el uso de beta-bloqueadores en el SCA por consumo de cocaína (26-28).

La importancia de los anteriores casos radica en que los tres son pacientes jóvenes y no tienen un uso de cocaína muy cercano al evento cardiaco. Es en este periodo temprano (inferior a dos horas) donde más se han descrito estas complicaciones. En nuestro conocimiento, en la literatura colombiana no se han descrito series de casos de pacientes jóvenes con infarto por cocaína, por lo cual nuestro reporte contribuye al conocimiento de esta complicación. El IAM asociado a consumo de cocaína es más frecuentemente descrito en hombres y siempre se debe considerar la cardiotoxicidad de la cocaína y la ruptura asociada de una placa (no solo el vaso espasmo como causa del dolor).

Todos los casos descritos son coherentes con lo reportado en la literatura a nivel mundial. Se encuentran otros factores de riesgo como el tabaquismo y los infartos son de tipo I, lo que evidencia la cardio toxicidad de la cocaína. Con respecto al primer caso, donde hay un diagnóstico de hipertensión previo, vale la pena resaltar a la cocaína como una causa de hipertensión en pacientes jóvenes.

Los anteriores casos, en comparación a otros reportadoss en la literatura latinoamericana como el de Rener en el 2014 (11), muestran como uno de los motivos de consulta frecuente en el servicio de urgencia es el dolor torácico.

\section{CONCLUSIÓN}

El consumo de cocaína es una de las posibles etiologías del síndrome coronario agudo, sobre todo en los pacientes 
jóvenes. Antes de la terapia de reperfusión, se debe considerar el rol de las benzodiacepinas en estos pacientes, insistiendo en su uso temprano. Estos pacientes deben recibir manejo anticoagulante (con heparina no fraccionada o de bajo peso molecular), antiagregación, nitratos, estatinas y, en cuanto sea prudente, IECA y beta-bloqueador.

\section{REFERENCIAS}

1. Oficina de las Naciones Unidas contra la Droga y el Delito. Informe Mundial sobre las Drogas 2017 . 2017. 36 p. [citado 30 de diciembre de 2019]. Disponible en: https://www.unodc.org/wdr2017/field/WDR_Booklet1_ Exsum_Spanish.pdf

2. Botero EG, Ramírez CM, Rosero MP, Augusto H, Romero I. Reportes de drogas Colombia. 2017. 224 p. [citado 30 de diciembre de 2019]. Disponible en: http://www.odc. gov.co/Portals/1/publicaciones/pdf/odc-libro-blanco/ reporte_drogas_colombia_2017.pdf

3. Nguyen P, Kamran H, Nasir S, Chan W, Shah T, Deswal A, Bozkurt B. Comparison of Frequency of Cardiovascular Events and Mortality in Patients With Heart Failure Using Versus Not Using Cocaine. Am J Cardiol. 2017;119(12):2030-4. https://doi.org/10.1016/j. amjcard.2017.03.034

4. Kim ST, Park T. Acute and Chronic Effects of Cocaine on Cardiovascular Health. Int J Mol Sci. 2019 Jan 29;20(3):584. https://doi.org/10.3390/ijms20030584

5. Aquaro GD, Gabutti A, Meini M, Prontera C, Pasanisi E, Passino C, Emdin M,Lombardi M. Silent myocardial damage in cocaine addicts. Heart. 2011;97(24):2056-62. https://doi.org/10.1136/hrt.2011.226977

6. Keskin M, Hayıroğlu Mi̇, Keskin Ü, Kaya A, Aydın BA, Börklü EB, Çinier G, Eren M. The most dangerous complication of intranasal cocaine abuse in a young man:cardiac arrest. Am J Emerg Med. 2016;34(8):1731. e5-7. https://doi.org/10.1016/j.ajem.2015.12.043

7. Dean JH, Woznicki EM, O'Gara P, Montgomery DG, Trimarchi S, Myrmel T, Pyeritz RE, Harris KM, Suzuki T, Braverman AC, Hughes GC, Kline-Rogers E, Nienaber CA, Isselbacher EM, Eagle KA, Bossone E. Cocaine-related aortic dissection: lessons from the International Registry of Acute Aortic Dissection. Am J Med. 2014;127(9):878-85. https://doi.org/10.1016/j.amjmed.2014.05.005

8. Kumar S, Sanchez L, Srinivasamurthy R, Mathias PF. Cocaine-InducedElectrocardiographic Phenomenon. Tex Heart Inst J. 2018;45(4):273-4. https://doi.org/10.14503/ THIJ-18-6722

9. Winhusen T, Theobald J, Kaelber DC, Lewis D. The association between regular cocaine use, with and without tobacco co-use, and adverse cardiovascular and respiratory outcomes. Drug Alcohol Depend. $2020 ; 214: 108136$. https://doi.org/10.1016/j. drugalcdep.2020.108136

10. Hoffman R. Cocaine in: Flomenbaum NE, Goldfrank LR; Hoffman RS; Howland MaA, Lewin NA, Nelson LS: Goldfrank's toxicologic emergencies. 11th Ed. New York: MacGraw-Hill; 2011. pp 1133-46

11. Rener Andrés, Figueroa Sebastián. Efectos cardiovasculares de la cocaína: A propósito de dos casos. Rev.Urug.Cardiol. 2014; 29(1):60-6.

12. McCord J, Jneid H, Hollander JE, de Lemos JA, Cercek B, Hsue P, Gibler WB,Ohman EM, Drew B, Philippides G, Newby LK; American Heart Association Acute Cardiac Care Committee of the Council on Clinical Cardiology. Management of cocaine-associated chest pain and myocardial infarction: a scientific statement from the American Heart Association Acute Cardiac Care Committee of the Council on Clinical Cardiology. Circulation. 2008;117(14):1897-907. https://doi. org/10.1161/CIRCULATIONAHA.107.188950

13. Cheng YC, Ryan KA, Qadwai SA, Shah J, Sparks MJ, Wozniak MA, Stern BJ, Phipps MS, Cronin CA, Magder LS, Cole JW, Kittner SJ. Cocaine Use and Risk of IschemicStroke in Young Adults. Stroke. 2016;47(4):91822. https://doi.org/10.1161/STROKEAHA.115.011417

14. Siniscalchi A, De Sarro G, Pacifici R, Pisani E, Sanguigni S, Gallelli L. Thrombolytic Therapy in Cocaine Users with Ischemic Stroke: A Review of Current Practice. Psychopharmacol Bull. 2019;49(1):70-9.

15. DeFilippis EM, Singh A, Divakaran S, Gupta A, Collins BL, Biery D, et al. Cocaine and Marijuana Use Among Young Adults With Myocardial Infarction. J Am Coll Cardiol. 2018 ;71(22):2540-51. https://doi.org/10.1016/j. jacc.2018.02.047

16. Nogué S, Picón M, Mestre G, Corcuera R. Urgencias en usuarios de cocaína. Med Integral, 39 (2002), pp. 249-59.

17. Callaghan RC, Halliday M, Gatley J, Sykes J, Taylor L, Benny C, Kish SJ. Comparative hazards of acute myocardial infarction among hospitalized patients with methamphetamine- or cocaine-use disorders: A retrospective cohort study. Drug Alcohol Depend. 2018;188:259-65. https://doi.org/10.1016/j. drugalcdep.2018.03.030

18. Morales-Bustamante JF, Berrouet-Mejía MC. Cocaína y estado convulsivo. Rev CES Med. 2012; 26(2): 215-21.

19. Schwartz BG, Rezkalla S, Kloner RA. Cardiovascular effects of Cocaine Circulation. 2010; 122 (24): 2558-2569. https://doi.org/10.1161/CIRCULATIONAHA.110.940569

20. O'Leary ME, Hancox JC. Role of voltage-gated sodium, potassium and calcium channels in the development 
of cocaine-associated cardiac arrhythmias. Br J Clin Pharmacol. 2010;69(5):427-42. https://doi.org/10.1111/ j.1365-2125.2010.03629.x

21. Ifedili I, Bob-Manuel T, Kadire SR, Heard B, John LA, Zambetti B, Heckle MR,thomas F, Haji S, Khouzam RN, Reed GL, Ibebuogu UN. Cocaine Positivity in ST-Elevation Myocardial Infarction: A True or False Association. Perm J. 2019;23.

22. Phang KW, Wood A. Cocaine use and delayed myocardial ischaemia and/or infarction. BMJ Case Rep. 2014;2014. https://doi.org/10.1136/bcr-2014-204599

23. Rezkalla SH, Kloner RA. Cocaine-induced acute myocardial infarction. Clin Med Res. 2007;5(3):172-6. https://doi.org/10.3121/cmr.2007.759

24. Khan R, Arshed S, Jehangir W, Sen S, Yousif A. CocaineInduced Delayed

25. Myocardial Infarction Complicated by Apical Thrombus. J Clin Med Res. 2016;8(1):59-61. https://doi.org/10.14740/ jocmr $2412 \mathrm{w}$

26. Riley ED, Hsue PY, Vittinghoff E, Wu AH, Coffin PO, Moore PK, Lynch KL. Higher prevalence of detectable troponin I among cocaine-users without known cardiovascular disease. Drug Alcohol Depend. 2017;172:88-93 https:// doi.org/10.1016/j.drugalcdep.2016.11.039

27. Pham D, Addison D, Kayani W, Misra A, Jneid H, Resar $\mathrm{J}$, Lakkis N, Alam M.Outcomes of beta blocker use in cocaine-associated chest pain: a meta-analysis.Emerg Med J. 2018;35(9):559-563. https://doi.org/10.1136/ emermed-2017-207065

28. Damodaran S. Cocaine and beta-blockers: the paradigm. Eur J Intern Med. 2010;21(2):84-6. https://doi. org/10.1016/j.ejim.2009.11.010

29. Cediel G, Carrillo X, García-García C, Rueda F, Oliveras T, Labata C, Serra J,Ferrer M, de Diego 0, Bayés-Genís A. $\beta$-Blocker treatment and prognosis in acutecoronary syndrome associated with cocaine consumption: The RUTI-Cocaine Study.Int J Cardiol. 2018; 260:7-10. https:// doi.org/10.1016/j.ijcard.2018.02.013 